\title{
Dor musculoesquelética em mulheres da indústria do vestuário: análise na perspectiva do modelo demanda-controle*
}

\section{Musculoskeletal pain in women of the clothing industry: analysis through the perspective of the demand-control model}

\author{
Viviane Gontijo Augusto $^{1}$, Cecília Ferreira Aquino², Daniel Silva Penha Gontijo ${ }^{3}$, \\ Rosana Ferreira Sampaio ${ }^{4}$
}

http://dx.doi.org/10.11606/issn.2238-6149.v27i2p172-179

\begin{abstract}
Augusto VG, Aquino CF, Gontijo DSP, Sampaio RF. Dor musculoesquelética em mulheres da indústria do vestuário: análise na perspectiva do modelo demanda-controle. Rev Ter Ocup Univ São Paulo. 2016 maio-ago.;27(2):172-9.
\end{abstract}

RESUMO: O objetivo do estudo foi descrever a ocorrência de dor musculoesquelética (DME) em trabalhadoras da indústria do vestuário e analisar a relação entre DME e diferentes formas de operacionalizar a variável "alta exigência psicossocial". Trezentas e seis trabalhadoras de 40 empresas foram avaliadas em relação à DME, através do auto-relato sobre a presença ou não do sintoma. Para avaliação dos fatores psicossociais adotou-se o Job Content Questionnaire (JCQ). A partir dos escores do JCQ, a variável "alta exigência psicossocial" foi operacionalizada nas formas: quadrante, razão, logaritmo da razão e subtração. Foram testados os modelos de regressão de Poisson, considerando cada forma de cálculo da variável exposição e tendo como desfecho a DME. Das trabalhadoras avaliadas, 54,3\% apresentavam DME. A probabilidade de ocorrência de DME na presença de alta exigência no trabalho variou de $1,2 \%$ a $75 \%$, de acordo com a operacionalização desta variável. A partir dos resultados obtidos, pode-se concluir que situações de trabalho com alta exigência psicossocial estão associadas com dores musculoesqueléticas em trabalhadoras da indústria do vestuário. Entretanto, a forma de definição e operacionalização da variável exposição (alta exigência) interfere nos valores de probabilidade de ocorrência de dor.

DESCRITORES: Esgotamento profissional; Recursos humanos; Trabalho feminino/psicologia; Mulheres/psicologia.
Augusto VG, Aquino CF, Gontijo DSP, Sampaio RF. Musculoskeletal pain in women of the clothing industry: analysis through the perspective of the demand-control model. Rev Ter Ocup Univ São Paulo. 20162016 May-Aug.;27(2):172-9.

\begin{abstract}
The objective of the study was to describe the occurrence of musculoskeletal pain (MEP) in workers of the clothing industry and analyze the relationship between MEP and different forms of operationalizing the variable "high psychosocial requirement". Three hundred and six workers from 40 companies were evaluated for MEP, through a self-report on the presence or absence of symptoms. For evaluating the psychosocial factors, we used the Job Content Questionnaire (JCQ). Through the JCQ scores, the "high psychosocial requirement" variable was operationalized in forms: quadrant, ratio, logarithm of the ratio and subtraction. We tested the Poisson regression models, considering every form of calculation of the exposure variable and having MEP as the outcome. Of the workers evaluated, $54.3 \%$ had MEP. The probability of MEP occurrence in the presence of high requirement at work in this study ranged from $1.2 \%$ to $75 \%$, according to the operationalization of this variable. With the results obtained, we concluded that work situations with high psychosocial requirements are associated with musculoskeletal pain in workers of the clothing industry. However, the form of definition and operationalization of the exposure variable (high requirement) interfere in the values of probability of pain occurring.
\end{abstract}

KEYWORDS: Musculoskeletal pain; Occupational exposure; Textile industry.

*Os dados do presente estudo fazem parte do projeto de doutorado da primeira autora, intitulado "Interação de fatores psicossociais e a capacidade para o trabalho em trabalhadoras da indústria do vestuário".

1. Doutora em Ciências da Reabilitação pela Universidade Federal de Minas Gerais (UFMG). Professora do curso de Fisioterapia da Universidade Estadual de Minas Gerais (UEMG), integrante do Núcleo de Saúde Coletiva, Divinópolis, Minas Gerais Brasil. Email: vaugusto@divinopolisuemg.com.br

2. Mestre em Ciências da Reabilitação pela Universidade Federal de Minas Gerais (UFMG). Professora do curso de Fisioterapia da Universidade Estadual de Minas Gerais (UEMG), integrante do Núcleo de Saúde Coletiva, Divinópolis, Minas Gerais Brasil. Email: ceciliafaquino@gmail.com

3. Doutor em Patologia Geral (UFTM), Professor do curso de Fisioterapia da Universidade Estadual de Minas Gerais (UEMG), integrante do Núcleo de Saúde Coletiva, Divinópolis, Minas Gerais Brasil. Email: danielsilva999@yahoo.com.br

4. Doutora em Saúde Publica pela Universidade Autônoma de Barcelona (UAB). Professora do programa de Pós - Graduação em Ciências da Reabilitação (UFMG) Belo Horizonte, Minas Gerais Brasil. Email: rosana.sampaio@pq.cnpq.br

Endereço para correspondência: Viviane Gontijo Augusto. Rua Planalto, 210, Bairro Manoel Valinhas. Divinópolis, MG, Brasil. CEP: $35500-281$. 


\section{INTRODUÇÃO}

s dores musculoesqueléticas representam
um grave problema para o trabalhador,
que se sente debilitado para realizar suas tarefas, e para a empresa, que sofre as consequências da diminuição na produtividade e dos custos de licenças e afastamentos ${ }^{1,2}$. Dados da previdência social indicam que as dores musculoesqueléticas representam a terceira causa de afastamento do trabalho no Brasil, o que tem provocado uma crescente busca por serviços médicos e de reabilitação ${ }^{3}$.

Os gastos médicos e sociais que se originam desse problema têm crescido nos últimos anos, impactando de modo significativo o orçamento da saúde pública e remetendo à necessidade de se identificar os fatores causais, para que sejam criadas estratégias de prevenção deste agravo ${ }^{4}$.

A relação entre dores musculoesqueléticas e fatores psicossociais do trabalho tem sido estudada em diversas categorias profissionais, embora a mensuração destes fatores ainda represente um desafio para os pesquisadores $^{2,4,5}$. Estes fatores englobam aspectos como excesso de tarefas, pressão de tempo e repetitividade; bem como monotonia, baixa demanda e falta de criatividade. Também são considerados aspectos psicossociais, o poder de decisão do trabalhador sobre o seu trabalho, conflitos interpessoais e falta de apoio social ${ }^{4}$.

Os fatores psicossociais são investigados a partir de alguns modelos teóricos, como o proposto por Karasek et al. ${ }^{6}$ em 1979, conhecido como Modelo Demanda Controle (DC). Este modelo foi desenvolvido em um contexto histórico onde a tendência emergente na Europa e Estados Unidos era enfatizar o poder e controle do trabalhador no ambiente de trabalho. Assim, o modelo demanda controle concebe duas dimensões que abrangem aspectos específicos do processo de trabalho ${ }^{6}$. O modelo DC se ancora na premissa de que o desequilíbrio da relação entre as demandas psicológicas no trabalho e a habilidade efetiva do trabalhador no enfrentamento destas demandas pode gerar estresse ocupacional ${ }^{7}$.

Neste modelo, demandas psicológicas se referem ao quanto este é excessivo e difícil de ser realizado, bem como à quantidade de conflitos existentes nas relações de trabalho. Por outro lado, controle é definido como a amplitude ou margem de decisão que o trabalhador possui em relação a dois aspectos: autonomia para tomar decisões sobre seu próprio trabalho e possibilidade de ser criativo e de adquirir novos conhecimentos ${ }^{7}$. O principal pressuposto do Modelo DC é que a condição de maior exposição ao adoecimento está nos trabalhos que apresentam alta exigência psicossocial ${ }^{8}$ (Figura 1).

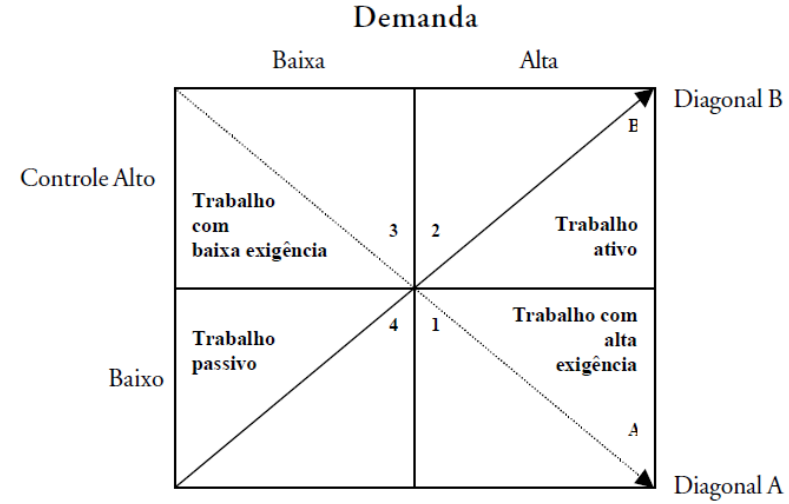

Fonte: Karasek \& Theörell ${ }^{6}$

Figura 1 - Representação gráfica do modelo Demanda-Controle

Evidências têm demonstrado que trabalhos com alta exigência psicossocial apresentam maior chance de repercutir negativamente na saúde ${ }^{8,9}$. Entretanto, ainda não há consenso com relação a melhor forma de se definir e operacionalizar este construto. Uma revisão sistemática sobre a utilização empírica do Modelo DC mostrou que a heterogeneidade na operacionalização da variável alta exigência psicossocial e nos parâmetros adotados para se definir alta exigência, pode estar contribuindo para os resultados tão distintos encontrados nos estudos que analisaram a relação entre estresse ocupacional e outros desfechos de saúde ${ }^{10}$.

$\mathrm{Na}$ vida adulta, os determinantes sociais de saúde, em sua maioria, estão conectados com a vida no trabalho ${ }^{11}$. Além disso, estes determinantes são distribuídos de forma desigual entre classes ocupacionais e entre homens e mulheres ${ }^{12,13}$. As mulheres são maioria em classes ocupacionais de menor renda e, frequentemente, o estresse ocupacional está ligado a trabalhos tradicionalmente considerados femininos como educação, cuidado de enfermos e costura ${ }^{14}$. Diferenças de gênero relacionadas às condições de emprego têm impacto na saúde e segurança ocupacional e não podem ser ignoradas.

As trabalhadoras do setor do vestuário são afetadas por diferentes condições de saúde como hipertensão arterial, distúrbios psíquicos, visuais e auditivos e agravos de origem musculoesquelética ${ }^{13}$. Embora muitos sintomas estejam associados aos distúrbios musculoesqueléticos relacionados ao trabalho, uma das queixas mais prevalentes é a dor ${ }^{12}$ As dores musculoesqueléticas são descritas como um desfecho de saúde comum entre professores ${ }^{13}$, enfermeiro ${ }^{12}$ e trabalhadoras da indústria do vestuário ${ }^{15}$.

Considerada polo da indústria do vestuário, a cidade de Divinópolis, localizada na região centro-oeste de Minas 
Gerais, mostra uma predominância de micro e pequenas empresas que, aliada à baixa qualificação da força de trabalho, contribui para a exploração e discriminação das mulheres, que representam cerca de $90 \%$ dos trabalhadores destas indústrias ${ }^{15}$. Assim, investigar a ocorrência de dores musculoesqueléticas nas trabalhadoras da indústria do vestuário e testar a sua associação com fatores psicossociais poderá auxiliar na definição de estratégias preventivas e de promoção da saúde dessa população. Além disto, considerando a importância do Modelo DC para a saúde do trabalhador e o seu uso no Brasil, faz-se necessário analisar as distintas formas de se operacionalizar o constructo alta exigência no trabalho.

Deste modo, tendo como referência o Modelo DC, os objetivos desse estudo foram descrever a ocorrência de dor musculoesquelética em trabalhadoras da indústria do vestuário e analisar a relação entre diferentes formas de se operacionalizar alta exigência no trabalho e dor musculoesquelética nesta população.

\section{MÉTODOS}

Trata-se de um estudo observacional transversal, cujos dados fazem parte do projeto intitulado Interação de fatores psicossociais e a capacidade para o trabalho em trabalhadoras da indústria do vestuário. Estes dados foram coletados de janeiro a dezembro de 2011, com participação de 306 trabalhadoras de 40 indústrias do vestuário de micro e pequeno porte do município de Divinópolis, Minas Gerais. O estudo foi aprovado pelo Comitê de Ética da Universidade Federal de Minas Gerais (UFMG), sob o parecer ETIC 0196.0.203.000-10. A amostra foi selecionada por conveniência. $\mathrm{O}$ contato inicial com as empresas foi feito por telefone e após assinatura do termo de anuência pelas empresas, iniciou-se a coleta de dados. Um único pesquisador coletou os dados, em horário previamente estabelecido, dentro da própria indústria. Todas as trabalhadoras foram convidadas a participar do estudo, permanecendo aquelas que concordaram e assinaram o Termo de Consentimento Livre e Esclarecido. A coleta durou cerca de 20 minutos para cada trabalhadora.

Para descrição da amostra foi utilizado um protocolo de avaliação contendo questões sociodemográficas (idade, escolaridade, estado civil, número de filhos) e questões relativas ao trabalho, tais como, setor, tempo e horário de trabalho na empresa. Estes dados faziam parte de um protocolo de avaliação elaborado pelos próprios autores, contendo apenas questões fechadas.

Para a avaliação dos fatores psicossociais adotouse o Job Content Questionnaire (JCQ), desenvolvido a partir do Modelo $\mathrm{DC}^{6}$. A validade e confiabilidade da versão traduzida para o português deste questionário foi realizada por Araújo e Karasek ${ }^{16}$. O JCQ é um instrumento que permite a avaliação do estresse no trabalho com base na relação entre demanda psicológica e controle no processo laboral. O instrumento contém 49 questões. Para cada questão, há uma escala que varia de 1 a 4 pontos ( $1=$ discordo fortemente; $2=$ discordo; $3=$ concordo; $4=$ concordo fortemente). A partir desta pontuação é possível calcular separadamente os escores para demanda psicológica e controle no trabalho. Estes escores podem ser utilizados na forma contínua ou pela divisão em quantis (tercil e quartil). A definição da variável independente "alta exigência psicossocial" pode ser feita de diversas maneiras, a partir da utilização destes escores. Neste estudo, optou-se pelas formas mais utilizadas na literatura, conforme apontado na revisão sistemática realizada por Alves et al. ${ }^{17}$. São elas: quadrante, razão (contínua, tercil e quartil), logaritmo da razão (contínua, tercil e quartil) e subtração (contínua, tercil e quartil).

Para a operacionalização na forma quadrante, inicialmente, as variáveis contínuas, demanda psicológica e controle, foram categorizadas, considerando como ponto de corte a mediana (abaixo da mediana = baixo; acima da mediana $=$ alto), resultando em quadrantes, conforme descrição: baixa exigência (alto controle e baixa demanda), alta exigência (baixo controle e alta demanda), trabalho ativo (alto controle e alta demanda), trabalho passivo (baixo controle e baixa demanda).

Para as demais formas de operacionalização foram calculados os valores contínuos da razão (razão entre demanda e controle), do logaritmo da razão, e por fim, da forma subtração (diferença entre demanda e controle). Estas três formas foram também categorizadas em alta exigência quando os valores foram superiores ao $2^{\circ}$ tercil - percentil 66 (razão, logaritmo da razão e subtração tercil); ou superiores ao $3^{\circ}$ quartil - percentil 75 (razão, logaritmo da razão e subtração quartil).

A dor musculoesquelética foi avaliada pelo autorelato da trabalhadora sobre a presença ou não de dor musculoesquelética. Em caso positivo, a trabalhadora deveria informar qual o local da dor.

A análise descritiva das diversas formas de se operacionalizar alta exigência foi realizada e, em seguida, foram testados os modelos de regressão de Poisson com variância robusta, considerando cada forma de cálculo da variável exposição e tendo como desfecho a ocorrência de dor musculoesquelética. Estes modelos são indicados em estudos de corte transversal com desfechos binários (e.g. ocorrência ou não de dor), sendo a associação entre 
exposição e desfecho estimada pela razão de prevalência (RP). Para cada modelo, neste estudo, foram estimados os valores de Razão de Prevalência (RP) com seus respectivos Intervalos de Confiança de 95\% (IC95\%).

Todas as análises foram realizadas com o software STATA versão 10.0, considerando um nível de significância de $5 \%$.

\section{RESULTADOS}

\section{Dados descritivos}

Participaram do estudo 306 trabalhadoras com média de idade de 36,02 anos $(\mathrm{DP}=11,07)$. Quanto à escolaridade, $22,6 \%$ tinham ensino fundamental incompleto, $37,6 \%$ haviam cursado o ensino fundamental completo ou médio incompleto e 39,8\% tinham ensino médio completo ou mais.

Quanto ao estado civil e número de filhos, observouse que pouco mais da metade da amostra $(58,2 \%)$ vivia com companheiro e $60,5 \%$ tinham filhos, sendo que $46,0 \%$ destas tinham até dois filhos. As 306 trabalhadoras estavam assim distribuídas nos setores produtivos: $62,5 \%$ no setor de costura, $27,6 \%$ na passadoria e $9,9 \%$ no acabamento. Em todas as empresas, a jornada de trabalho era de nove horas diárias (das 7 às 17h15), com pausa de uma hora para almoço e 15 minutos para café, de segunda à quintafeira. Na sexta-feira, o horário de trabalho era das 7 às 16 h para completar as 44 horas semanais. Ressalta-se que $31,7 \%$ das trabalhadoras faziam hora extra. O tempo de trabalho na indústria variou de um (1) a 324 meses com mediana de 33 meses ou 2,75 anos.

Entre as trabalhadoras avaliadas, $166(54,3 \%)$ apresentavam dor musculoesquelética. As regiões corporais mais acometidas foram: coluna $(21,9 \%)$, braços/ ombros $(12,0 \%)$, joelhos/pernas $(8,5 \%)$, mãos $(4,7 \%)$, cabeça $(4,3 \%)$ e outros locais $(2,9 \%)$.

\section{Dados do questionário JCQ}

A análise isolada dos itens do JCQ relativos às dimensões demanda psicológica e controle no trabalho, permitiu observar que: com relação a variável controle, o trabalho exige um alto nível de habilidade para a maioria das trabalhadoras $(87,6 \%)$, mas elas têm oportunidade de aprender coisas novas (81\%). Ainda sobre o controle no trabalho, as decisões sobre as tarefas foram menos frequentes $(28,8 \%)$ (Tabela 1$)$.

As características que mais se destacaram em relação à alta demanda psicossocial foram a exigência de longos períodos de concentração nas tarefas $(85,6 \%)$, seguido de trabalho repetitivo $(84,0 \%)$ (Tabela 1$)$.

Tabela 1 - Descrição das características das dimensões Controle e Demanda psicológica para trabalhadoras da indústria do vestuário. Divinópolis, Minas Gerais, $2011(\mathrm{~N}=306)$

\begin{tabular}{l|c|c|c|c}
\hline \multirow{2}{*}{ Características do controle } & \multicolumn{2}{|c|}{ Não } & \multicolumn{2}{c}{ Sim } \\
\cline { 2 - 5 } & $\mathbf{N}$ & $\mathbf{\%}$ & $\mathbf{N}$ & $\mathbf{\%}$ \\
\hline Aprender coisas novas & 58 & 19,0 & 248 & 81,0 \\
\hline Requer criatividade & 124 & 40,6 & 182 & 59,4 \\
\hline Exige alto nível de habilidade & 38 & 12,4 & 268 & 87,6 \\
\hline Encarregado de tarefas diferentes & 138 & 45,1 & 168 & 54,9 \\
\hline $\begin{array}{l}\text { Oportunidade de desenvolver } \\
\text { tarefas especiais }\end{array}$ & 95 & 31,0 & 211 & 69,0 \\
\hline Tomar decisões sobre tarefas & 218 & 71,2 & 88 & 28,8 \\
\hline Pouca liberdade para decidir & 140 & 45,7 & 166 & 54,3 \\
\hline Características de demanda & & & & \\
\hline Trabalho repetitivo & 49 & 16,0 & 257 & 84,0 \\
\hline Ritmo acelerado & 113 & 40,0 & 193 & 60,0 \\
\hline $\begin{array}{l}\text { Não tenho volume excessivo de } \\
\text { trabalho }\end{array}$ & 130 & 42,4 & 176 & 57,6 \\
\hline $\begin{array}{l}\text { Exige longos períodos de } \\
\text { concentração nas tarefas }\end{array}$ & 44 & 14,4 & 262 & 85,6 \\
\hline As tarefas são interrompidas & 120 & 39,2 & 186 & 60,8 \\
\hline O trabalho é frenético & 210 & 68,7 & 96 & 31,3 \\
\hline $\begin{array}{l}\text { Esperar pelo trabalho dos outros } \\
\text { torna mais lento o trabalho }\end{array}$ & 148 & 48,4 & 158 & 51,6 \\
\hline $\begin{array}{l}\text { Tempo para realizar a tarefa é } \\
\text { suficiente }\end{array}$ & 42 & 13,8 & 264 & 86,2 \\
\hline Livre de demandas conflitantes & 150 & 49,1 & 156 & 50,9 \\
\hline
\end{tabular}

Após analisar a variável alta exigência no trabalho operacionalizada nas formas descritas na metodologia deste estudo observa-se na Tabela 2 que a prevalência de alta exigência variou de $24,8 \%$ (forma do quadrante) a $36,9 \%$ (forma da subtração tercil) (Tabela 2).

Tabela 2 - Prevalência de alta exigência de acordo com a forma de operacionalização da variável - Trabalhadoras da indústria do vestuário, Divinópolis, Minas Gerais, 2011 (N=306)

\begin{tabular}{l|c|c}
\hline Formas de operacionalizar & Frequência & Percentual \\
\hline Quadrante & & \\
\hline baixa exigência & 92 & 30,1 \\
\hline alta exigência & 76 & 24,8 \\
\hline trabalho ativo & 87 & 28,4 \\
\hline trabalho passivo & 51 & 16,7 \\
\hline \multicolumn{2}{|c}{ Continua... }
\end{tabular}


Augusto VG, et al. Dor musculoesquelética em mulheres da indústria do vestuário. Rev Ter Ocup Univ São Paulo. 2016 maio/ago.;27(2):172-9.

Tabela 2 - Prevalência de alta exigência de acordo com a forma de operacionalização da variável - Trabalhadoras da indústria do vestuário, Divinópolis, Minas Gerais, $2011(\mathrm{~N}=306)$

\begin{tabular}{l|c|c}
\hline Formas de operacionalizar & Frequência & Percentual \\
\hline Razão Tercil & & \\
\hline baixa exigência & 196 & 64,1 \\
\hline alta exigência & 110 & 35,9 \\
\hline Razão Quartil & 230 & \\
\hline baixa exigência & 76 & 24,8 \\
\hline alta exigência & & \\
\hline Logaritmo da Razão Tercil & 196 & 64,1 \\
\hline baixa exigência & 110 & 35,9 \\
\hline alta exigência & & \\
\hline Logaritmo da Razão Quartil & 230 & 75,2 \\
\hline baixa exigência & 76 & 24,8 \\
\hline alta exigência & & \\
\hline Subtração Tercil & 193 & 63,1 \\
\hline baixa exigência & 113 & 36,9 \\
\hline alta exigência & & \\
\hline Subtração Quartil & 222 & 72,5 \\
\hline baixa exigência & 84 & 27,5 \\
\hline alta exigência & &
\end{tabular}

\section{Análise de regressão de Poisson}

A análise da associação entre alta exigência no trabalho e dor musculoesquelética pelo modelo de Poisson indicou que um indivíduo com alta exigência no trabalho, segundo a operacionalização na forma quadrante, tem uma probabilidade $44 \%$ maior de ter dor musculoesquelética que aquele exposto à baixa exigência $(p=0,013)$.

Os resultados mostraram associações entre as variáveis ao se utilizar a operacionalização na forma razão contínua $(\mathrm{p}=0,010)$ e razão quartil $(\mathrm{p}=0,037)$. No caso da razão contínua, o aumento de uma unidade no valor de alta exigência aumentou em $75 \%$ a probabilidade de ocorrência de dor. Para a razão quartil, um indivíduo com alta exigência no trabalho, tem uma probabilidade $25 \%$ maior de apresentar dor musculoesquelética comparado àqueles com baixa exigência.

Também houve associação entre alta exigência e dor musculoesquelética para as formas logaritmo da razão contínua e logaritmo da razão quartil $(\mathrm{p}=0,014 \mathrm{e}$ 0,037 , respectivamente). No caso do logaritmo da razão contínua, o aumento de uma unidade para alta exigência aumentou em $53 \%$ a probabilidade de ocorrência de dor. Para o logaritmo da razão quartil, um indivíduo submetido à alta exigência no trabalho tem uma probabilidade $25 \%$ maior de dor que aquele com baixa exigência.
A forma subtração mostrou associação entre alta exigência psicossocial e presença de dor musculoesquelética apenas para a variável subtração contínua $(\mathrm{p}=0,01)$. $\mathrm{O}$ aumento de uma unidade no valor da subtração contínua aumentou em 1,2\% a probabilidade de ocorrência de dor musculoesquelética (Tabela 3).

Tabela 3 - Modelo de Poisson com variância robusta considerando diferentes formas de operacionalização da variável de exposição (alta exigência psicossocial) e presença de Dor Musculoesquelética como desfecho para trabalhadoras da indústria do vestuário. Divinópolis, Minas Gerais, $2011(\mathrm{~N}=306)$

\begin{tabular}{l|c|c|c}
\hline Operacionalização & Valor-p & RP & IC 95\% \\
\hline Quadrante & & & \\
\hline baixa exigência & - & 1,00 & \\
\hline alta exigência & $\mathbf{0 , 0 1 3}$ & 1,44 & {$[1,08 ; 1,91]$} \\
\hline trabalho ativo & 0,100 & 1,28 & {$[0,95 ; 1,71]$} \\
\hline trabalho passivo & 0,328 & 1,19 & {$[0,84 ; 1,68]$} \\
\hline Razão Contínua & $\mathbf{0 , 0 1 0}$ & 1,75 & {$[1,14 ; 2,67]$} \\
\hline Razão Tercil & & & \\
\hline alta exigência & 0,350 & 1,10 & {$[0,90 ; 1,36]$} \\
\hline Razão Quartil & & & \\
\hline alta exigência & $\mathbf{0 , 0 3 7}$ & 1,25 & {$[1,01 ; 1,55]$} \\
\hline $\begin{array}{l}\text { Logaritmo da Ra- } \\
\text { zão Contínua }\end{array}$ & $\mathbf{0 , 0 1 4}$ & 1,53 & {$[1,09 ; 2,14]$} \\
\hline $\begin{array}{l}\text { Logaritmo da } \\
\text { Razão Tercil }\end{array}$ & & & \\
\hline alta exigência & 0,350 & 1,11 & {$[0,90 ; 1,36]$} \\
\hline $\begin{array}{l}\text { Logaritmo da } \\
\text { Razão Quartil }\end{array}$ & & & \\
\hline alta exigência & $\mathbf{0 , 0 3 7}$ & 1,25 & {$[1,01 ; 1,55]$} \\
\hline $\begin{array}{l}\text { Subtração Contí- } \\
\text { nua }\end{array}$ & $\mathbf{0 , 0 1 0}$ & 1,012 & {$[1,002 ; 1,021]$} \\
\hline Subtração Tercil & & & {$[0,91 ; 1,37]$} \\
\hline alta exigência & 0,308 & 1,11 & \\
\hline $\begin{array}{l}\text { Subtração Quartil } \\
\text { alta exigência }\end{array}$ & 0,059 & 1,23 & {$[0,99 ; 1,51]$} \\
\hline RP= Razão de Prevalência & IC95\% $]$ \\
\hline
\end{tabular}

\section{DISCUSSÃO}

Os resultados do presente estudo evidenciaram que a probabilidade de ocorrência de dor musculoesquelética na presença de alta exigência no trabalho variou de acordo com a forma de operacionalização desta variável. A probabilidade mais elevada foi encontrada na forma razão contínua $(75 \%) \mathrm{e}$ a menor probabilidade na forma subtração (1,2\%). De acordo 
com Alves et al. ${ }^{17}$, a forma razão não parece ser adequada uma vez que é possível se obter o mesmo resultado na divisão dos números em situações diferentes. Dito de outra forma, números distintos podem expressar razões semelhantes, embora as situações de demanda e controle sejam diferentes. Contrapondo a esta argumentação, Campos ${ }^{18}$ a partir da análise de cortisol salivar de trabalhadores da enfermagem, considerou a forma subtração como a mais adequada para discriminar alta e baixa exigência no trabalho.

Neste estudo, dores musculoesqueléticas associaramse com todas as formas utilizadas para operacionalização da variável alta exigência psicossocial e as evidências disponíveis atualmente ainda são insuficientes para que seja estabelecido se uma destas formas é superior às outras ${ }^{19}$, mas é importante destacar que a maneira de se computar esta variável pode influenciar na sua interpretação. Nas abordagens de razão, logaritmo e subtração é possível identificar um trabalho que apresente alta exigência psicossocial mesmo em situações de trabalho com baixa demanda e alto controle, diferentemente da abordagem quadrante que considera esta situação como um trabalho de baixa exigência. Por outro lado, o pressuposto do Modelo DC, na operacionalização da forma quadrante, é que trabalhos com alta demanda e alto controle devem ser considerados como trabalhos ativos, ou seja, trabalhos que proporcionam oportunidade de aprendizado e aprimoramento de habilidades. Assim, o que determina se o trabalho é estressante ou não é o nível de controle do trabalhador sobre as atividades desempenhadas ${ }^{7}$. Porém, do ponto de vista de saúde do trabalhador, isto se torna perigoso, pois pode haver situações de sobrecarga, com consequências negativas para a saúde, devido ao excesso de demanda, mesmo na presença de alto controle. Esta questão já foi apontada em outros estudos que utilizaram o Modelo de Karasek e obtiveram evidências de que o alto controle no trabalho não foi efetivo na redução dos efeitos da alta demanda psicológica para a saúde dos trabalhadores ${ }^{16,20}$.

A amostra deste estudo foi constituída em sua maioria por mulheres jovens, cujo tempo de trabalho na empresa apresentou grande variabilidade, com mediana de aproximadamente três anos. Estes aspectos deveriam contribuir para um número reduzido de dores musculoesqueléticas e de estresse devido à baixa idade e o pequeno tempo de exposição à tarefa. Apesar do pequeno tempo de trabalho na empresa, mais da metade das trabalhadoras deste estudo apresentou dores musculoesqueléticas, e estas foram apontadas em diferentes regiões corporais, sendo mais frequentes as queixas na região da coluna vertebral. Este resultado corrobora o estudo de Andersen et al..$^{21}$ que relataram que a prevalência de dores musculoesqueléticas em mais de uma região anatômica é comum em atividades com movimentos repetidos e acelerados e entre aqueles que trabalham, na maior parte do tempo, sentados. A análise do processo de trabalho realizada por Augusto et al. ${ }^{15}$ mostrou que as trabalhadoras permanecem fixas em seus postos de trabalho durante toda a jornada, sejam sentadas, como no setor de costura e acabamentos, ou de pé, nos setores de corte e passadoria. Além disso, para obter uma melhor visualização durante a realização das tarefas, as trabalhadoras avaliadas pelos pesquisadores mantinham uma postura de flexão da coluna cervical e não raramente adotavam a postura de inclinação e rotação de tronco.

A manutenção da postura sentada durante longas jornadas de trabalho contribui para o desenvolvimento de disfunções associadas aos sistemas vascular e musculoesquelético ${ }^{22,23}$. A posição sentada gera compressão de vasos sanguíneos, ocasionando dores em membros inferiores, edemas nos pés e sensação de fadiga nas pernas ${ }^{22}$. A postura sentada, especialmente se o tronco é inclinado anteriormente e mantido em posição estática, também está associada a dores musculoesqueléticas, principalmente na região lombar ${ }^{23}$.

Para além de fatores biomecânicos, os aspectos ergonômicos e psicossociais também se associam com dores musculoesqueléticas ${ }^{1,2}$. Neste estudo, a análise dos itens do JCQ relativos às dimensões demanda psicológica e controle no trabalho mostrou características clássicas da organização científica do trabalho. Esta forma de organização desapropriou o saber operário e tornou as atividades repetitivas, monótonas e desestimulantes, além de exigir o máximo da capacidade física e psíquica dos trabalhadores. As trabalhadoras deste estudo são submetidas a um trabalho repetitivo, com ritmo acelerado, e que exige alta concentração na realização das tarefas. Estas características representam um processo de trabalho com alta demanda, que aliado a um baixo poder de decisão sobre as tarefas e principalmente sobre as políticas da empresa, coloca as trabalhadoras numa situação de exposição ao estresse.

Por se tratar de um estudo transversal não é possível inferir causalidade, ou seja, não se pode afirmar que as características do trabalho foram causas das dores musculoesqueléticas. Entretanto, foi possível demonstrar associação entre estas variáveis, independente da forma de operacionalização da variável de exposição (alta exigência). Estes resultados reforçam as evidências de que um trabalho estressante pode ser fonte de adoecimento físico e psicológico ${ }^{24}$.

Uma limitação do presente estudo é o possível efeito "trabalhador sadio", ou seja, é possível que o estudo tenha avaliado somente as trabalhadoras mais saudáveis, que 
estavam presentes na empresa no momento da pesquisa, uma vez que não se sabe quantas trabalhadoras podem ter se afastado do trabalho por motivo de doença. Também podem ter sido selecionadas empresas onde as condições de trabalho são menos desfavoráveis à saúde, pois a seleção das empresas foi por conveniência. Assim, pode ter havido uma subestimação tanto da variável desfecho quanto da variável de exposição.

Apesar das limitações mencionadas, o presente estudo contribui para o entendimento sobre a precariedade do trabalho das mulheres da indústria do vestuário. A presença de riscos biomecânicos e psicossociais indica a necessidade de ações educativas e medidas preventivas como orientação postural, mudanças de posição e adequação de mobiliários de trabalho. A introdução de pausas, revezamento de funções, maior poder de decisão sobre as tarefas e sobre o ritmo de trabalho também podem ajudar na adequação das condições de trabalho e na redução das dores musculoesqueléticas. A adoção de medidas preventivas traz, não só benefícios diretos para as trabalhadoras, mas também gera economia para o sistema previdenciário por evitar afastamentos e aposentadorias precoces.

\section{CONCLUSÃO}

O presente estudo contribuiu para a compreensão de que situações de trabalho com alta exigência psicossocial estão associadas com dores musculoesqueléticas em trabalhadoras da indústria do vestuário e que a forma de definição e operacionalização da variável exposição (alta exigência) interfere na probabilidade de ocorrência de dor. Neste estudo, a probabilidade mais alta de ocorrência de dor foi encontrada na forma razão contínua e a menor probabilidade na forma subtração de operacionalização da variável "alta exigência", com uma grande variabilidade entre estes valores $(1,2 \%$ para a forma subtração e $75 \%$ para a forma razão contínua). Futuras pesquisas que utilizem avaliações mais objetivas do estresse em trabalhadores podem auxiliar na definição de parâmetros para se operacionalizar a alta exigência psicossocial no trabalho, facilitando a comparação de resultados entre os estudos e o entendimento sobre como esta característica dos trabalhadores pode se associar com desfechos negativos de saúde.

Os resultados evidenciaram a necessidade de reflexão sobre o impacto das condições de trabalho, da rigidez organizacional e da perda de controle sobre a atividade laboral, na saúde das trabalhadoras da indústria do vestuário. Assim, o empoderamento dessas mulheres, por meio de oficinas e processos educativos contínuos, pode favorecer para que elas assumam o controle no enfrentamento das condições de trabalho e ainda pode facilitar adoção de medidas preventivas que reduzam dores musculoesqueléticas e possam evitar afastamentos e aposentadorias precoces neste setor.

Contribuições dos autores: V. G. Augusto - participou da concepção, coleta de dados e redação final do manuscrito; C. F. Aquino participou da análise de dados e redação final do manuscrito; D. S. P. Gontijo - participou da análise de dados e redação final do manuscrito; R. F. Sampaio - participou da concepção e redação final do manuscrito.

\section{REFERÊNCIAS}

1. Magnago TSBS, Lisboa MTL, Griep RH, Zeitoune RCG, Tavares JP. Distúrbios musculo-esqueléticos em trabalhadores de enfermagem: associação com condições de trabalho. Rev Bras Enferm. 2007;60(6):701-5. DOI: 10.1590/ S0034-71672007000600015.

2. Cardoso PJ,Araujo MT, Carvalho MF, Oliveira FN, Reis BFJE. Aspectos psicossociais do trabalho e dor musculoesquelética em professores. Cad Saúde Pública. 2011;27(8):1498-506. DOI: $10.1590 / \mathrm{S} 0102-311 \mathrm{X} 2011000800005$.

3. Brasil. Ministério da Previdência Social. Anuário estatístico da previdência social - AEPS 2007. Brasília: MPS/ DATAPREV; 2008. Disponível em: http://programamineracao.
org.br/wp-content/uploads/2011/08/Anu\%C3\%A1rioEstat\%C3\%ADstico-da-Previd\%C3\%AAncia-Social-2007.pdf.

4. Sampaio RF, Coelho CM, Barbosa FB, Mancini MC, Parreira VF. Work ability and stress in a bus transportation company in Belo Horizonte, Brazil. Cienc Saude Coletiva. 2009;14(1):287-96. DOI: 10.1590/S141381232009000100035 .

5. Karasek RA. Job demand, job decision latitude, and mental strain: implications for job redesign. Admin Sci Q. 1979;24:285-308. DOI: 10.2307/2392498.

6. Karasek RA, Theorell T. Healthy work. Stress, productivity and the reconstruction of working life. New York: Basic Books; 1990. 
7. Greco PBT, Magnago TSBS, Beck CLC, Urbanetto JS, Prochnow A. Estresse no trabalho em agentes dos centros de atendimento sócio educativo do Rio Grande do Sul. Rev Gaúcha Enferm. 2013;34(1):94-103. DOI: 10.1590/S198314472013000100012 .

8. Alcântara MA, Sampaio RF, Souza, M, Silva FCM, Kirkwood RN. Chronic pain profile: an interaction between biological and psychosocial factors. Pain Studies Treat. 2013;1(2):9-16. DOI: $10.4236 /$ pst.2013.12003.

9. Alves MGM, Hökerberg YHM, Faerstein E. Tendências e diversidade na utilização empírica do Modelo DemandaControle de Karasek (estresse no trabalho): uma revisão sistemática. Rev Bras Epidemiol. 2013;16(1):125-36. DOI: 10.1590/S1415-790X2013000100012.

10. Buss PM, Pellegrini Filho A. A saúde e seus determinantes sociais. Physis. 2007;17(1):77-93. DOI: 10.1590/S010373312007000100006 .

11. Tinubu BM, Mbada CE, Oyeyemi AL, Fabunmi AA. Work-related musculoskeletal disorders among nurses in Ibadan, South-west Nigeria: a cross-sectional survey. BMC Musculoskelet Disord. 2010;11:12. DOI: 10.1186/14712474-11-12.

12. Toivanen S. Social determinants of stroke as related to stress at work among workingwomen: a literature review. Stroke Res Treat. 2012;ID873678. DOI: 10.1155/2012/873678.

13. Maciel ACC, Fernandes MB, Medeiros LS. Prevalência e fatoresassociadosàsintomatologiadolorosa entreprofissionais da indústria têxtil. Rev Bras Epidemiol. 2006;9(1):94-102. DOI: $10.1590 / \mathrm{S} 1415-790 X 2006000100012$.

14. Augusto VG, Sampaio RF, Ferreira FR, Kirkwood RN, César CC. Factors associated with inadequate work ability among women in the clothing industry. Work. 2015;50(2):275-83. DOI: 10.3233/WOR-131801.

15. Araújo TM, Karasek R. Validity and reliability of the job content questionnaire informal and informal jobs in Brazil. Scand J Work Environ Health Suppl. 2008;34(6):52-9.

16. Alves MGM, Braga MV, Faerstein E, Lopes SC, Junger W. Modelo demanda-controle de estresse no trabalho: considerações sobre diferentes formas de operacionalizar a variável de exposição. Cad Saude Publica. 2015;31(1):20812. DOI: $10.1590 / 0102-311 X 00080714$.

17. Campos JF. Estresse ocupacional segundo o modelo demandacontrole e suas repercussões na saúde do trabalhador da Enfermagem: análise das variações do cortisol salivar [Tese]. Rio de Janeiro: Faculdade de Enfermagem, Universidade do Estado do Rio de Janeiro; 2013. Disponível em: http://www. bdtd.uerj.br/tde_busca/arquivo.php?codArquivo=5201.

18. Courvoisier DS, Perneger TV. Validation of alternative formulations of job strain. J Occup Health. 2010;52(1):5-13. DOI: org/10.1539/joh.L9084.

19. Urbanetto JS, Magalhães MCC, Maciel VO, Santanna VM, Gustavo AS, Figueiredo CEP, Magnago TSBS. Estresse no trabalho segundo o Modelo Demanda-Controle e distúrbios psíquicos menores em trabalhadores de enfermagem. Rev Esc Enferm USP. 2013;47(5):1180-6. DOI: 10.1590/S0080623420130000500024

20. Andersen JH, Haahr JP, Frost P. Risk factors for more severe regional musculoskeletal symptoms: a two years prospective study of a general working population. Arthritis Rheum. 2007;56(4):1355-64.

21. Krijnen RMA, De Boer EM, Bruynzeel DP. Epidemiology of venous disorders in the general and occupational populations. Epidemiol Rev. 1997;19(2):294-309. DOI: 10.1016/j. ijnurstu.2011.09.005.

22. Claus AP, Hides JD, Moseley GL, Hodges PW. Is 'ideal' sitting posture real? Measurements of spinal curvatures in four sitting postures. Man Ther. 2009;14(4):404-8. DOI: 10.1016/j.math.2008.06.001.

23. Griep RH, Rotenberg L, Landsbergis P, Vasconcellos-Silva PR. Uso combinado de modelos de estresse no trabalho e a saúde auto-referida na enfermagem. Uso combinado de modelos de estresse no trabalho e a saúde auto-referida na enfermagem. Rev Saude Publica. 2011;45(1):145-52. DOI: 10.1590/S0034-89102011000100017.

Recebido em: 01.10.15

Aceito em: 18.04 .16 